\title{
A Field Experiment Demonstrating Plant Life-History Evolution and Its Eco-Evolutionary Feedback to Seed Predator Populations
}

\author{
Anurag A. Agrawal, ${ }^{1,2, \star}$ Marc T. J. Johnson, ${ }^{3}$ Amy P. Hastings, ${ }^{1}$ and John L. Maron ${ }^{4}$ \\ 1. Department of Ecology and Evolutionary Biology, Cornell University, Ithaca, New York 14853; 2. Department of Entomology, \\ Cornell University, Ithaca, New York 14853; 3. Department of Biology, University of Toronto at Mississauga, Mississauga, Ontario L5L \\ 1C6, Canada; 4. Division of Biological Sciences, University of Montana, Missoula, Montana 59812
}

Online enhancement: appendix. Dryad data: http://dx.doi.org/10.5061/dryad.7h8n3.

\begin{abstract}
The extent to which evolutionary change occurs in a predictable manner under field conditions and how evolutionary changes feed back to influence ecological dynamics are fundamental, yet unresolved, questions. To address these issues, we established eight replicate populations of native common evening primrose (Oenothera biennis). Each population was planted with 18 genotypes in identical frequency. By tracking genotype frequencies with microsatellite DNA markers over the subsequent three years (up to three generations, $\approx 5,000$ genotyped plants), we show rapid and consistent evolution of two heritable plant life-history traits (shorter life span and later flowering time). This rapid evolution was only partially the result of differential seed production; genotypic variation in seed germination also contributed to the observed evolutionary response. Since evening primrose genotypes exhibited heritable variation for resistance to insect herbivores, which was related to flowering time, we predicted that evolutionary changes in genotype frequencies would feed back to influence populations of a seed predator moth that specializes on O. biennis. By the conclusion of the experiment, variation in the genotypic composition among our eight replicate field populations was highly predictive of moth abundance. These results demonstrate how rapid evolution in field populations of a native plant can influence ecological interactions.
\end{abstract}

Keywords: field experiment, flowering phenology, longevity, natural selection, plant resistance, plant-herbivore interactions, rapid evolution.

\section{Introduction}

Studies employing experimental evolution are a cornerstone of modern evolutionary biology (Garland and Rose 2009). They can directly test evolutionary theory, shed light on how different selective regimes shape evolutionary dynamics, and determine whether evolutionary trajectories

* Corresponding author; e-mail: agrawal@cornell.edu.

Am. Nat. 2013. Vol. 181, pp. S35-S45. (C) 2012 by The University of Chicago. 0003-0147/2013/181S1-53442\$15.00. All rights reserved.

DOI: $10.1086 / 666727$ occur in parallel across replicate populations (Reznick et al. 1990; Meyer and Kassen 2007; Blount et al. 2008). With field studies increasingly providing evidence of contemporary evolution (Kinnison and Hendry 2001; Maron et al. 2004; Grant and Grant 2008), it is becoming clear that there is strong potential for rapid evolutionary dynamics to influence ecological interactions over short time spans (Hairston et al. 2005).

Traditionally, evolutionary ecologists have used a "retrospective approach" to determine how current ecological dynamics may be influenced by the evolutionary history (i.e., phylogenetic relatedness) of community members (Losos 1994; Cavender-Bares et al. 2004; Mooney et al. 2010) or have asked how selective factors that differ between populations may have influenced trait differentiation (Carroll and Boyd 1992; Brodie et al. 2002; Smith and Benkman 2007). An alternative but powerful approach involves quantifying how contemporary evolutionary change in the field influences ecological dynamics. This still rarely used approach can shed light on feedbacks between rapid evolution and ecology (Hersch-Green et al. 2011). However, most field experiments aimed at assessing the effect of evolution on ecological dynamics typically begin with genetically differentiated populations rather than monitoring evolutionary change over time (Harmon et al. 2009; Palkovacs and Post 2009; Bassar et al. 2010). We know of no multigenerational field experiments that have quantified the extended ecological consequences of evolutionary change on other community members as evolution has actually happened in the field (Schoener 2011). Accordingly, we examined evolution in replicated populations with an initially identical genetic structure and monitored the consequences for community dynamics.

In addition to shedding light on feedbacks between evolutionary dynamics and ecological processes, field experiments quantifying rapid evolution can be used to address 
several other important issues. First, to what extent do replicate populations experiencing similar environmental conditions show parallel evolutionary trajectories? When populations repeatedly evolve in the same direction and at the same rate (i.e., parallel evolution), this suggests that selection outweighs contingency and stochastic factors (e.g., genetic drift) and therefore that evolutionary dynamics are predictable. Second, to what extent does fecundity predict which genotypes are favored over time? Many of our best case studies of selection-in-action are single-generation analyses of the fitness associated with particular genes or genotypes, but the evolutionary response to this selection is often unknown (Barrett et al. 2008; Morrissey et al. 2010). Multigenerational experiments account for the sometimes hidden life-history transitions that can be critical for the response to natural selection.

We conducted a field experiment where we quantified the evolution of life-history traits of common evening primrose (Oenothera biennis) in replicated populations containing identical genotypes planted in a resource-rich environment with low competition. Theory predicts the evolution of shorter life spans (i.e., annuality) in such high-quality environments (Hart 1977; Law et al. 1977; Lacey et al. 1983), and our base population varied considerably for this heritable life-history trait (Johnson et al. 2009a). We also determined whether differential lifetime seed production accurately predicted evolutionary responses or whether other demographic attributes (i.e., germination rates) contributed to realized fitness. Finally, we explored how evolutionary changes in our experimental plant populations affected ecological dynamics of an abundant insect herbivore. Specifically, we tested the ability to predict the abundance of a specialist seed predator moth on the basis of evolved changes in genotype frequencies, and thus heritable plant traits, of our replicate populations. Such a demonstration of how evolutionary change could modulate an ecological interaction would be strong evidence for an eco-evolutionary feedback (Schoener 2011).

\section{Biology of Oenothera biennis Relevant to Experimental Evolution}

Common evening primrose O. biennis L. (Onagraceae) is an herbaceous plant native to open habitats in eastern North America (fig. 1). Plants typically germinate in spring, form a rosette, bolt and flower at the end of the first (annual) or second (biennial) growing season, and immediately die after reproduction (i.e., they are semelparous or monocarpic). We have repeatedly observed high heritability for both life span (annual vs. biennial) and flowering phenology among our genotypes (table A1, available online; Johnson and Agrawal 2005; Johnson et al. 2009a). In previous single-generation experiments, we found natural selection on plant life-history traits and evidence that life-history traits were important predictors of insect attack (Johnson and Agrawal 2005; Johnson 2007; Johnson et al. 2009b).

Oenothera biennis is almost exclusively self-fertilizing, and its genetic system prevents recombination or segregation of alleles, resulting in seeds that are typically genetically identical to each other and to their maternal parent (Cleland 1972; Rauwolf et al. 2008; Johnson 2011). Because plants self-fertilize and are semelparous, seed production represents an important component of lifetime male and female fitness. Seed dispersal occurs passively by gravity, with no apparent traits that promote long-distance movement. We took advantage of this dispersal behavior and genetic system in O. biennis to construct eight replicate populations, each composed of the same 18 distinct genotypes distinguished by microsatellite markers (Larson et al. 2008). An important aspect of this study is that $O$. biennis's reproductive system makes it possible to study evolutionary change within populations according to changes in genotype frequencies through time and to relate this evolution to changes in life-history traits. Furthermore, by tracking the frequency of individual genotypes, we were able to relate reproductive measures (i.e., lifetime seed production) to realized fitness, filling a gap that is well known but has been difficult to bridge over multiple generations (Harper 1977; Ehrlén 2003; Waser et al. 2010).

In this study, we specifically focus on three life-history traits: life span (annual vs. biennial), flowering phenology, and seed emergence. We measured the first two traits, which varied substantially among genotypes, directly in the experimental populations (table A1; see also "Methods"). We measured seed emergence in a separate experiment under natural field conditions: the genotypes showed considerable variation in percent emergence (germination followed by establishment), which ranged 17fold across the 18 genotypes (table A1). These three traits (annuality, phenology, and emergence) were not genetically correlated (Pearson correlations on clonal means: $n=18, r$ values all positive but $<0.15$, and all $P>.50$ ).

\section{Methods}

Field Experiment

In May 2007, we established eight experimental populations in an abandoned agricultural field in Tompkins County, Ithaca, New York. Plots had an area of $13.5 \mathrm{~m}^{2}$ and were spaced at least $10 \mathrm{~m}$ apart from each other. The field encompassing the plots had homogeneous vegetation and shared an identical land use history (abandoned agriculture). They were plowed and sprayed twice with her- 

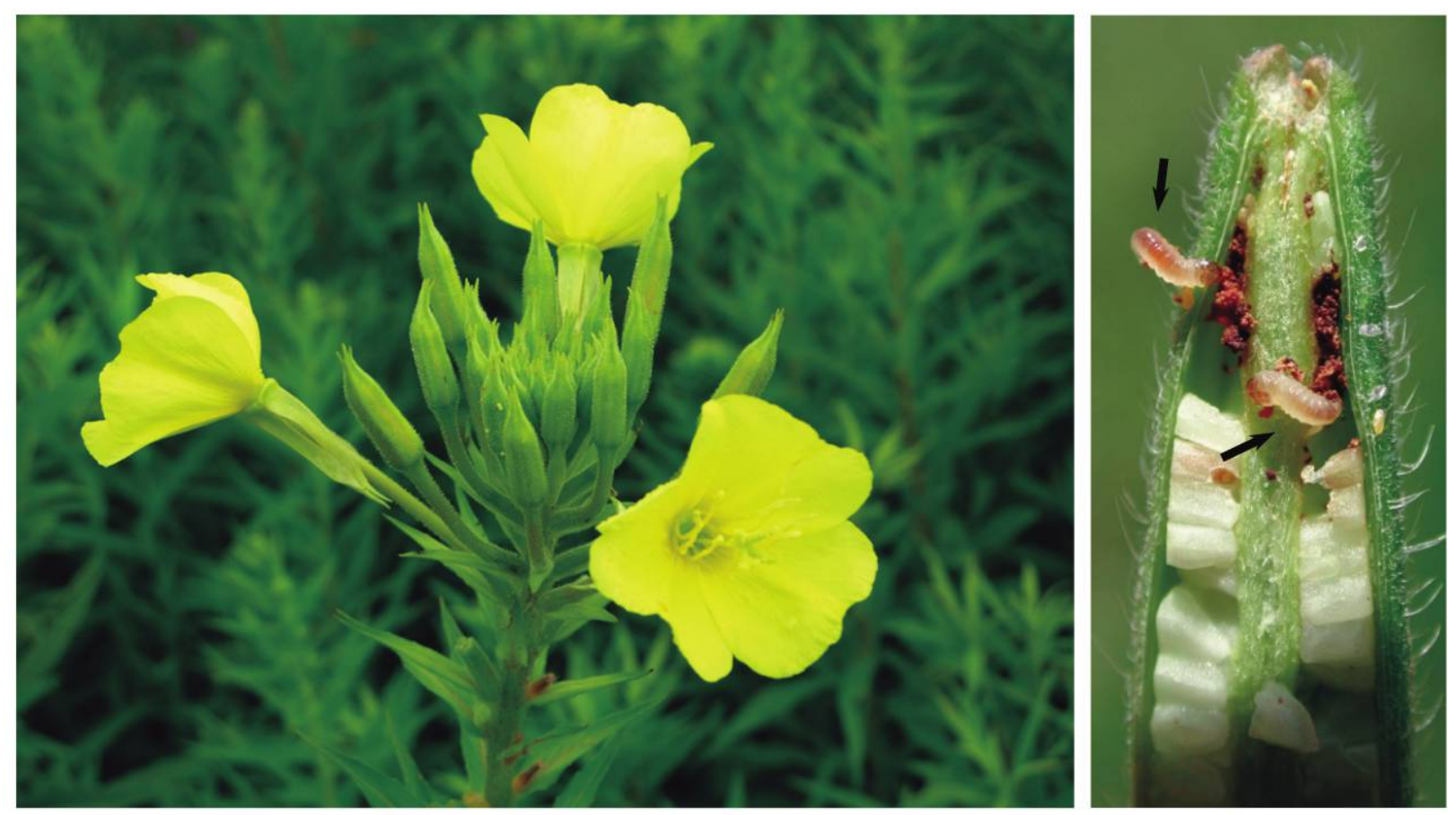

Figure 1: Common evening primrose (Oenothera biennis) growing in our experimental evolution plots near Ithaca, New York. The image on the right shows a cross section of a fruit, with two larvae (arrows) of Mompha brevivittella consuming immature (white) seeds; note the frass next to the larvae.

bicide before the experiment and were protected from deer herbivory by mesh fencing. At this highly productive site, initially with little plant competition, genotypes were up to twice as fecund as they were when planted at alternative sites in Tompkins County (A. A. Agrawal, unpublished data). In the center of each plot, we established a $1 \times 1$ $\mathrm{m}$ focal area that was planted with 60 individual Oenothera biennis seedlings at the first true leaf stage. Plants were equally spaced. There was no further manipulation of the plots: they were not weeded, and recruitment in subsequent years occurred throughout the $13.5-\mathrm{m}^{2}$ plot area. The nearest natural populations of $\mathrm{O}$. biennis were more than $1 \mathrm{~km}$ from our plots, and therefore the potential for outside contamination was low.

We transplanted three individuals from each of 16 genotypes and six individuals from each of two additional genotypes $(3 \times 16+6 \times 2=60$ total individuals per plot) into each plot, with the location of each genotype randomly assigned. The latter two genotypes were originally thought to be four distinct genotypes, but extensive additional analyses indicate that these were in fact indistinguishable (see details below in "Genotyping"). The 18 genotypes were selected to span the range of phenotypic characteristics from a total of 40 genotypes, which were all grown in a common garden in 2006 (Johnson et al. 2009a). The 40 original collections were all from earlysuccessional habitats (one per site, separated by at least $0.5 \mathrm{~km}$; average distance between sites, $12 \mathrm{~km}$, with a maximum of $30 \mathrm{~km}$; all from Tompkins County, NY) typical of the species (Johnson et al. 2009a). Although our field experiment was also located in Tompkins County, the closest collection site was $\approx 2 \mathrm{~km}$ away. To reduce the influence of maternal environment effects, we grew all genotypes for a generation in a common garden at the same site and used their offspring in our experiment.

In each year, we assessed seed production and recruitment. Populations of plants increased rapidly, with a mean $( \pm \mathrm{SE})$ of $3,476 \pm 694,4,167 \pm 625$, and $1,656 \pm 224$ plants per plot in 2008, 2009, and 2010, respectively. We counted fruits for all original 60 plants in each focal area (2007 and 2008). However, because of the large episode of recruitment beginning in 2008, we subsampled populations in each year between 2008 and 2010 by genotyping at least 190 individuals from each plot. We randomly sampled rosettes according to their proportion (relative to flowering plants) of the total population of recruits in each year. In total, we genotyped 4,931 plants between 2008 and 2010. In addition to all focal-area plants, at the end of each growing season we counted all fruits on at least 50 genotyped individuals per plot. We estimated seed pro- 
duction on the basis of genotypically variable mean fruit size $\left(F_{17,44}=3.61, P<.001\right)$ and the regression slope of fruit size (volume) and seed number $(Y=2.33 x-$ 102.47, $\left.r^{2}=0.60, F_{1,26}=38.47, P<.001\right)$.

\section{Seed Predators and Ecological Feedbacks of Evolutionary Change}

A specialist seed predator moth, Mompha brevivittella, was abundant in our plots. This native moth is univoltine and lays eggs on developing evening primrose fruits; larvae feed entirely inside the fruits on seeds, with one larva typically destroying $20 \%$ of the seeds per fruit. Each year, we estimated abundance of $M$. brevivittella on the basis of the number of emergence holes from 50 fruits from the same individual plants used to measure seed production. We estimated seed loss to herbivory on the basis of the correlation between seeds/fruit and the number of $M$. brevivittella per fruit. Genotypic differences in moth abundance from the beginning of the experiment were used to predict plot-level moth abundances across our eight replicate populations according to their respective genotype frequencies. In other words, we attempted to predict the ecological feedback of evolutionary change in the plant populations by tracking frequencies of genotypes and their known (genotypic) resistance to seed predators.

\section{Genotyping}

From each plant, we collected a leaf piece $\left(\approx 1 \mathrm{~cm}^{2}\right)$ directly into a 96-well plate on dry ice. Tissue was stored at $-80^{\circ} \mathrm{C}$ and then freeze-dried before DNA extraction. Freeze-dried tissue was ground to a fine powder in the collection plate with 3-mm stainless steel beads (in a mixer mill; Retsch, Haan, Germany), and for the majority of samples, genomic DNA was obtained by incubating samples at $60^{\circ} \mathrm{C}$ in a cetyltrimethylammonium bromide (CTAB) buffer containing betamercaptoethanol for $20 \mathrm{~min}$ and extracting the lysate with chloroform. DNA was precipitated from the aqueous phase with isopropanol, washed with ethanol, dried, and resuspended in Tris-EDTA buffer. For a portion of samples from $2008(\approx 550)$, DNA was extracted directly from freeze-dried tissue with the Extract-N-Amp Plant PCR Kit (Sigma-Aldrich, St. Louis, MO), according to the manufacturer's protocol. For each plate of extracted DNA, a subset of samples was analyzed for nucleic acid quantity and quality with a NanoDrop Spectrophotometer (Thermo Fisher Scientific, Waltham, MA). Entire plates were then diluted with water to yield approximate DNA concentrations of $5-20 \mathrm{ng} / \mu \mathrm{L}$.

For each sample, four microsatellite loci were amplified in a single multiplex PCR reaction (Type-It Microsatellite PCR kit; Qiagen, Valencia, CA). PCR reactions contained
$1 \mu \mathrm{L}$ of diluted genomic DNA and were set up according to the manufacturer's protocol (with Q-solution), but they were scaled to a $10-\mu \mathrm{L}$ total volume. Four pairs of primers were included in each reaction, with the forward primer for each locus being labeled with a distinct fluorescent tag (6-FAM, PET, NED, or VIC; Applied Biosystems, Life Technologies, Carlsbad, CA). Touchdown PCRs were performed with an initial activation at $95^{\circ} \mathrm{C}$ for $5 \mathrm{~min}$, followed by 34 cycles of $95^{\circ} \mathrm{C}$ for $30 \mathrm{~s}, 59^{\circ}-50^{\circ} \mathrm{C}$ for $90 \mathrm{~s}$ (the annealing temperature was decreased by $1^{\circ} \mathrm{C}$ in each of the first 10 cycles), and $72^{\circ} \mathrm{C}$ for $30 \mathrm{~s}$, and a final extension at $60^{\circ} \mathrm{C}$ for $30 \mathrm{~min}$. Multiplex PCR products were visualized for a subset of samples in each plate by means of agarose gel electrophoresis. Products were then diluted $1: 3$ with water and mixed with Hi-Di formamide and Genescan LIZ-500 size standard (Applied Biosystems). Four previously developed microsatellite loci (Larson et al. 2008) were used to differentiate genotypes, with the addition of one new marker that we developed to distinguish two particular genotypes (Oenbi102; forward primer: VIC-GAGAGGGCCAGATACGGAACAAT, reverse primer: GCAGAGCAGATAAAGGAGGGAGAG; Genbank accession no. JF825549). Approximately 380 samples from 2008 were genotyped for loci Oenbi2tri2, Oenbi2tri3, Oenbi2tri6, and Oenbi2tri7 (Larson et al. 2008), while Oenbi102 replaced Oenbi2tri7 in all subsequent multiplexing.

Samples were analyzed on a 3730xl DNA Analyzer (Applied Biosystems, Foster City, CA) at the Cornell University Life Sciences Core Laboratories Center. Allele sizes were determined with Genemapper software (ver. 3.5; Applied Biosystems), with all calls checked by eye. Plants were assigned to a particular genotype only if their alleles at all four loci were representative of that genotype. A subset of samples did not fall into known genotype categories and were presumed to be offspring of rare outcrossing events or, less likely, mutants or results of PCR error. These samples were excluded from analyses.

As mentioned above, what we initially thought to be four distinct genotypes (of 20) were actually two genotypes. This was confirmed by screening 27 additional microsatellite loci, which never distinguished the genotypes. We additionally used 454 Roche sequencing on all four genotypes and compared 163,000 sequence reads totaling 58 million bp of data between genotypes. Although insertion-deletion differences and single nucleotide polymorphisms were detected by 454 sequencing, subsequent attempts to validate these differences via Sanger sequencing showed that differences between genotypes were false positives. 


\section{Plant Traits}

We established an index of annuality by recording the proportion of the 24 individuals of each genotype that bolted in 2007. Although annuality in O. biennis can be somewhat plastic (Johnson 2007), our genotypic measure of annuality was robust to environmental conditions at the experimental site; in a separate common-garden study conducted with the same genotypes but under divergent growing conditions in 2006 (table A1; Johnson et al. $2009 a$ ), genotypic variation in annuality was consistent with our 2007 estimates $(n=18, r=0.60, P=.009)$. In particular, the growing environment in 2006 was highly competitive, with $O$. biennis being transplanted directly into meadow vegetation, compared to the 2007 planting on bare soil.

In July 2007 , when more than $25 \%$ of bolting plants had open flowers, we counted the number of open or senesced flowers (and ripening fruits) per plant (on the 384 plants that had bolted) as an index of phenology. Higher values indicate advanced phenology. Although this metric of phenology is not the typical measure (i.e., days until the first open flower), we believe that it is a robust measure for three reasons. First, because O. biennis produces relatively few flowers at a time and flowers last only for one night before senescing, the number of open and past flowers is a good indicator of phenological advancement. Second, this estimate is genetically correlated (negatively) with the onset of plant senescence (i.e., the termination of flowering; $n=18, r=-0.645, P=.004$ ), thus indicating that the window/duration of flowering is shifted among the genotypes. Finally, our measure of phenology from 2007 was genetically correlated with an independent measure of phenology from our experimental populations in 2010 ( $n=16$, because two genotypes were absent in 2010, $r=0.515, P=.041)$. Thus, although the environmental conditions were dramatically different across these years of measurement, our estimate of phenology was internally consistent across genotypes.

We estimated genotypic variation in emergence (potentially encompassing both dormancy and viability) in a separate experiment where we added 100 seeds of each genotype to replicated field plots $(n=5$ plots $\times 18$ genotypes). The 90 plots $(10 \mathrm{~cm} \times 10 \mathrm{~cm})$ were established on bare soil at the site of the main experiment in early December 2008, and percent emergence was assessed in April 2009. There was essentially no recruitment of foreign $O$. biennis outside of the seeds we sowed, as evidenced by a lack of emerging O. biennis on bare soil between or outside of the 90 experimental plots.

\section{Statistical Analyses}

We used repeated-measures canonical correspondence analysis to assess changes in genotypic structure (relative genotype frequencies) in the plots over time (Canoco for Windows, ver. 4.55). We assessed the statistical significance of consistent changes in genotypic structure using a Monte Carlo permutation test (Braak and Šmilauer 2002); the trace value, which is related to variation explained by the ordination axes, was tested across sampling dates with randomization (Braak and Šmilauer 2002). All other analyses involved genetic correlations (typically, $n=18$, conducted on genotypic means accounting for variation among the eight replicate plots) or ANOVAs (conducted in JMP, ver. 8, SAS Institute, Cary, NC). Unless transformations are indicated, data met the assumptions of the analyses.

The rate of predicted phenotypic evolution was estimated in terms of Haldanes, which were calculated as the absolute value of (estimated population mean at time 1 estimated population mean at time 0)/pooled standard deviation/generations (Kinnison and Hendry 2001). Standard deviations were pooled across the eight replicate populations, and each year was calculated as 0.72 generations, on the basis of the weighted average of annual and biennial life histories exhibited by plant genotypes in 2007 .

To assess the feedback of evolutionary change on seed predator populations, we conducted a regression analysis based on the replicate populations $(n=8)$. Here, we regressed the observed moth emergence data from 2010 (50 plants per plot) on the predicted moth populations (estimated by the product of the 2007 genotype-specific attack rates and the frequency of each genotype).

\section{Results}

We observed rapid evolution within all experimental populations. Genotype frequencies changed in a consistent manner across our eight replicate populations during three generations of population growth (repeated-measures canonical correspondence analysis: trace $=0.039, F=$ $3.085, P=.018$ ), with two genotypes consistently becoming extinct by 2010 and four genotypes increasing in frequency by two- to fourfold (fig. 2). Although there was variation among plots, as indicated by the error bars in figure 2, the statistically consistent decline in some genotypes and increase in others indicate that the observed evolutionary response was not overwhelmed by genetic drift (table A2, available online). This evolutionary change was also not the result of differential mortality in the first year (which was $<1 \%$ ) but was caused by differential recruitment over the subsequent three years. Populations evolved to have increased annuality and later flowering times (fig. 3). These evolutionary changes occurred rela- 


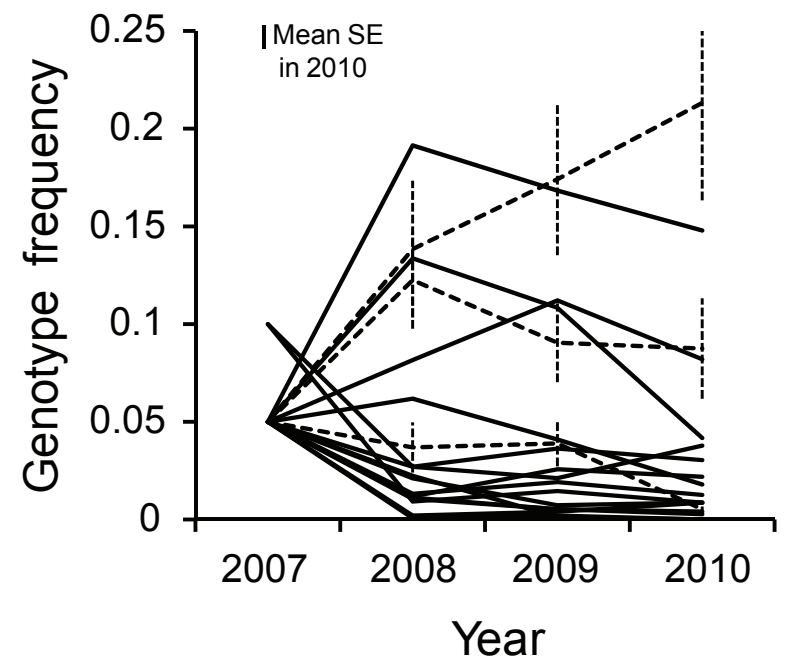

Figure 2: Changes in the frequencies of 18 genotypes of common evening primrose (Oenothera biennis) over three years after the start of the field experiment. Standard errors of the means are based on the eight replicate experimental populations and are shown for three arbitrary genotypes (dashed lines). Mean SE across all genotypes in 2010 was 0.01 .

tively rapidly, at rates of 0.34 and 0.21 Haldanes (change in units of standard deviations) in the first generation for annuality and phenology, respectively. Over the four years, the corresponding changes averaged 0.10 and 0.09 Haldanes per year for the two traits, indicating a substantially slower rate of evolutionary change after the first generation (fig. 3).

We took two approaches to identify which traits were favored by evolution. First, we calculated the genotypic covariance between the two life-history traits (annuality and flowering phenology) and two alternative measures of plant fitness (lifetime seed production and the product of emergence rate and lifetime seed production). Although there was substantial yearly variation in the strength of genotypic covariance between life-history traits and these two measures of fitness, both annuality and flowering phenology were significant predictors of actual changes in genotype frequencies over time, and this association was consistent across years (table 1; fig. 4). In other words, life-history traits did not always predict seed production, but they did predict the overall response to selection.

Second, we addressed the role of these alternative measures of plant fitness in predicting the changes in genotype frequencies. Genotypic differences in lifetime seed production, on their own, were not a significant predictor of subsequent genotype frequencies in 2008 or 2009 but were a significant predictor in 2010 (fig. A1, available online). Including emergence rate significantly improved our ability to predict evolutionary change only in 2008, when evolution was most rapid, and not in subsequent years (fig. A1). Thus, across the three years of our experiment, the primary contributors to fitness ranged from lifetime seed set to the product of this measure and emergence rate to neither of these proxies of fitness (fig. A1). In no case was cumulative seed production alone (summing across years) a strong predictor of subsequent genotype frequencies (all $P>.1$ ).

Eco-evolutionary feedbacks. Oenothera exhibited heritable variation for resistance to the specialist seed predator moth Mompha brevivittella, with broad-sense (clonal) heritabilities $\left(H^{2}\right)$ estimated as $0.14,0.55,0.33$, and 0.01 (all significantly different from 0 ) successively for the four years of the study. Based on changes in genotype frequencies (fig. 2) and this heritable resistance, the experimental populations increased in resistance across years (fig. A2, available online). Despite consistent overall evolutionary change in our experiment, our replicate popu-
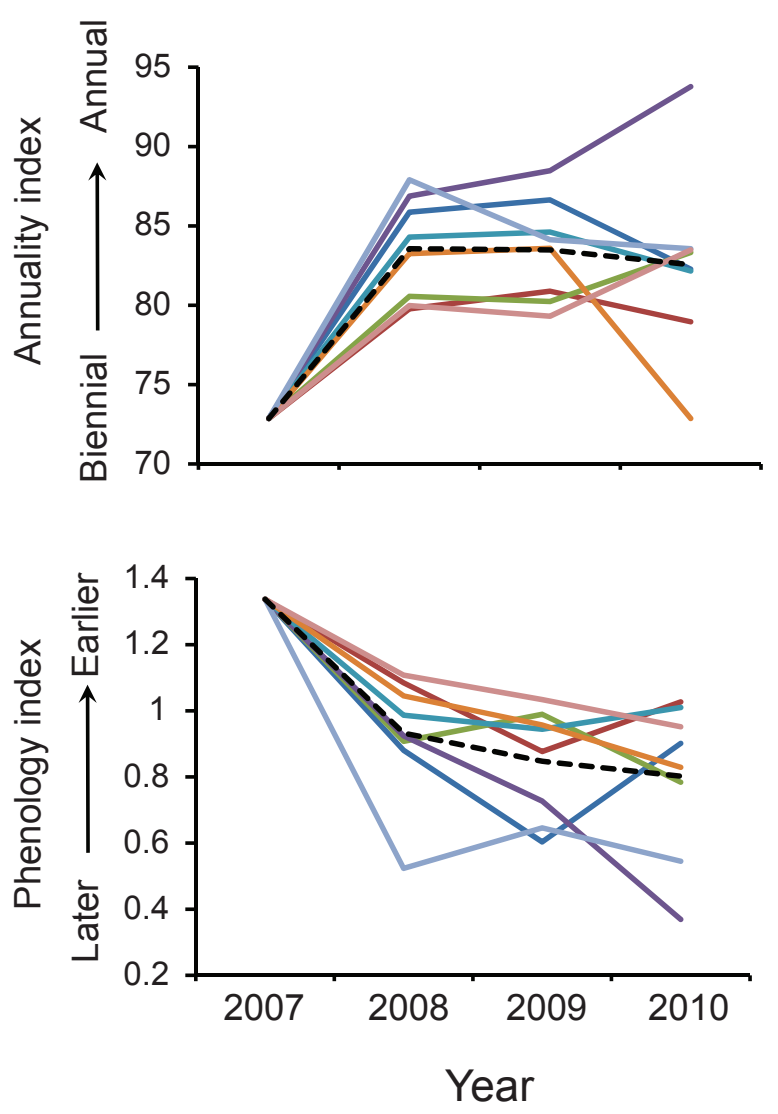

Figure 3: Estimates of the evolutionary change in two life-history traits of common evening primrose (Oenothera biennis) based on changes in genotype frequencies. Data are shown for each of the eight replicate experimental populations, with the mean represented by a dashed line. Genotype-specific values of life-history traits hold constant under different environmental conditions (see "Methods"). 
Table 1: Estimates of how two life-history traits (annuality and flowering phenology) predict seed production, germinable seeds (seed production $\times$ emergence rate), and observed evolution (genotype frequencies) in field plots of common evening primrose

\begin{tabular}{lcccc}
\hline & df & Seed production & Germinable seeds & $\begin{array}{c}\text { Realized genotype } \\
\text { frequency }\end{array}$ \\
\hline Annuality & 1,15 & 2.221 & 2.347 & $\mathbf{8 . 6 3 6}^{*}$ \\
Flowering phenology & 1,15 & $3.753^{\dagger}$ & 1.337 & $\mathbf{4 . 3 2 1}^{*}$ \\
Year & 2,14 & $\mathbf{1 0 . 2 3 5}^{* *}$ & $\mathbf{5 . 4 2 9}^{*}$ & .076 \\
Annuality $\times$ year & 2,14 & $\mathbf{1 6 . 5 9 3}^{* * *}$ & $\mathbf{9 . 3 3 8}^{* *}$ & .178 \\
Flowering phenology $\times$ year & 2,14 & .177 & $2.986^{\dagger}$ & .741 \\
\hline
\end{tabular}

Note: $F$ values are shown for three separate two-factor repeated-measures MANOVAs, with the plant traits as predictors over three years; full-model $r^{2}$ values for the three analyses are $0.527,0.437$, and 0.426 , respectively. Data are genotypic means $(n=18)$ averaged across the eight replicate populations. Values in boldface are statistically significant.

$\dagger P<.1$

* $P<.05$.

** $P<.01$.

*** $P<.001$.

lations showed some variation in genotypic structure and thus evolutionary trajectory. We took advantage of that variation among our eight experimental populations to examine whether there was an ecological feedback on populations of $M$. brevivittella. In other words, do replicate populations with greater representation of resistant genotypes produce fewer seed predator moths than populations that have lower frequencies of these same genotypes?

By the conclusion of the experiment in 2010, we found that differential changes in genotypic frequencies among our replicate populations predicted the actual abundance of $M$. brevivittella (fig. $5 ; n=8, r=0.908, P=.002$ ). This effect was not apparent in 2008 and 2009, the years in which heritability in resistance to $M$. brevivitella was greatest. This implies that the ecological consequences of evolution may be apparent only after several generations and only following the combined effects of selection and high heritability in the trait that has extended effects on the community. Genotype-specific attack rates were consistent between 2007 and 2010 (table A1; $n=16, r=$ $0.668, P=.005)$. More specifically, in 2010 flowering phenology predicted attack by $M$. brevivittella on the basis of phenotypic $(n=235, r=0.356, P<.001)$ and genetic ( $n=16, r=0.483, P=.003)$ correlations, while annuality did not. Because moths oviposit early in the flowering season and because at this stage our resistant genotypes have not yet begun flowering (table A1), these genotypes effectively escape seed predation. In particular, we typically observe oviposition by the three flower and seed predators (M. brevivittella, Mompha stellela, and Schinia florida) during the period when our experimental and natural populations are just beginning to flower (early July). Thus, the evolution of later-flowering phenology appears to be responsible for the ecological feedback to $M$. brevivittella.

\section{Discussion}

Across eight replicate populations, our field experiment demonstrated rapid and consistent genotypic changes over multiple generations, despite the potential for environmental variation or genetic drift to erode this pattern. Our results support the view that such evolutionary change in novel environments can be a potent driver of ecological dynamics. Specifically, consistent with life-history theory, Oenothera biennis populations rapidly evolved toward short, annual life cycles. In addition, the extent of this evolutionary change among our replicate populations significantly predicted changes in the abundance of a specialist seed predator moth. We discuss our results in light of these findings.

\section{Rapid Evolution of Plant Populations}

The observed rapid evolution of plant life-history traits was concordant with long-standing life-history theory (Charnov and Schaffer 1973) and our own previous singlegeneration analyses of natural selection on $O$. biennis (Johnson 2007). In particular, under high resources and low competition, selection is predicted to favor shorter life spans because of the positive multiplicative effects of low mortality and high fecundity for individuals that can develop and reproduce quickly, compared to individuals that delay development and reproduce at a later time (Hart 1977). However, we caution that this interpretation remains tentative because we have not directly compared life-history evolution in low- and high-resource environments. Law et al. (1977) reported that populations of Poa annua from low-competition environments had genetically based shorter life spans than populations from highcompetition environments. In contrast to our results, how- 

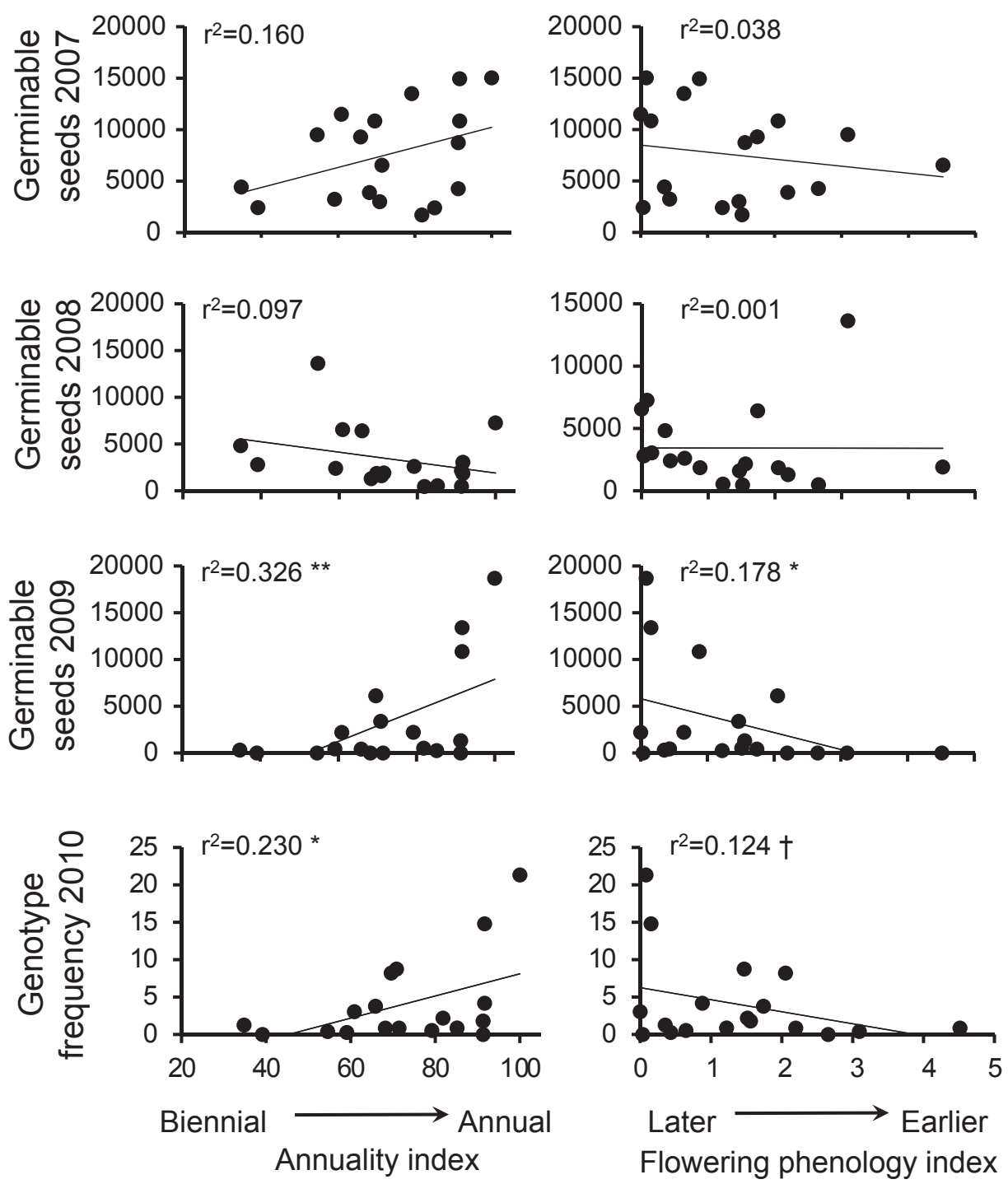

Figure 4: Relationship between genotype-specific values of two life-history traits and measures of genotypic fitness in 2007, 2008, and 2009 and of realized evolution in 2010. Data are genotypic means $(n=18)$ averaged across the eight replicate populations. The strength of natural selection varied across years (table 1). Raw data are shown, although statistical analyses and partial $r^{2}$ values are derived from multiple regression. A dagger $(\dagger)$ indicates $P<.1$, a single asterisk indicates $P<.05$, and a double asterisk indicates $P<.01$.

ever, Law et al. found that earlier flowering was also favored in low-competition environments. For O. biennis, selection consistently favored genotypes that flowered later (table 1), presumably because this allows for greater resource acquisition before the onset of reproduction and the avoidance of the specialist seed predator moth. Consistent with this conclusion, the earliest-flowering annuals had very low seed set.

The fact that annual genotypes quickly rose to prominence in year 2 (2008) is not surprising, because, by definition, annuals contributed all of the seed in the first year of the experiment. However, this purely demographic driver of genotypic change was not solely responsible for the ensuing evolutionary dynamics. For example, despite a huge pulse of seed input by biennial genotypes in the second year of the experiment, these genotypes remained underrepresented within plots in years 3 and 4 (2009 and 2010; table A1). In other words, seed production and realized genotype fitness were somewhat decoupled. We found a consistent advantage for annual life histories even though after year 2 , annual genotypes did not contribute the majority of seeds to plots. This result is consistent with 


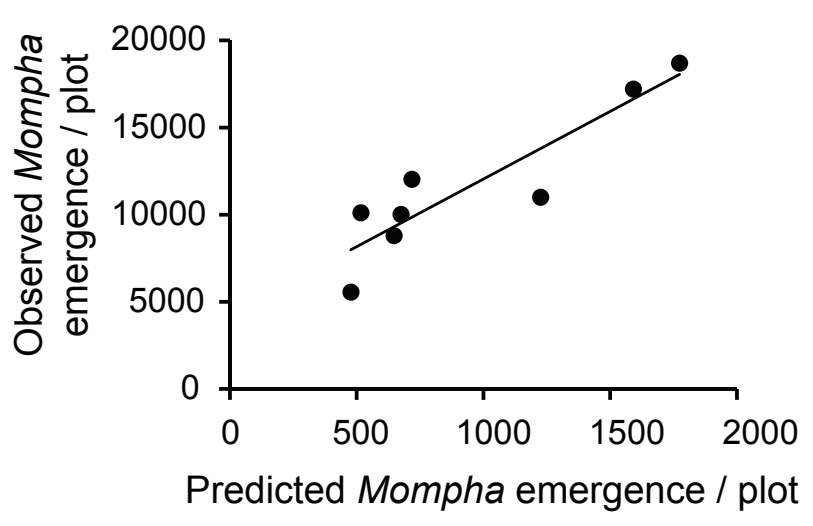

Figure 5: Feedback of evolution in common evening primrose (Oenothera biennis) populations to ecological dynamics of a specialist moth seed predator (Mompha brevivittella). Shown are data for the eight replicate plots; predicted seed predator abundances for 2010 were based on changes in genotype frequencies and genotype-specific attack rates measured in 2007.

previous measures of natural selection on this trait in the field, coupled with demographic models (Johnson 2007). Put another way, annuality consistently predicted genotype frequency in each year (i.e., no annuality $\times$ year interaction; table 1). This resulted from a complex mix of demographic effects (initial advantage of annuals), concurrent natural selection (on annuality and phenology), and likely altered environmental conditions (i.e., increasing competition and its effect on how annuality is expressed). In addition, flowering phenology showed continued evolutionary change over all years of the experiment (table 1; fig. 3), and this was not a by-product of changes in annuality, as the two traits were genetically uncorrelated.

As John Harper pointed out more than 30 years ago, lifetime seed production may not always predict plant fitness (Harper 1977), and recent demographic work on plant populations supports the notion that fecundity is only one component of fitness (Ehrlén 2003; Waser et al. 2010). In our analysis, including an additional demographic attribute (seedling emergence, which was likely driven by differences in dormancy) resulted in a substantial improvement of fitness estimates in the first year (2007). The maintenance of heritable variation in dormancy has been widely observed and was suggested as an adaptation to environmental heterogeneity (Jain 1982; Brown and Venable 1986). Nonetheless, genotype-specific seed emergence rates and seed production alone explained less than $6 \%$ of variation in genotype frequencies in the next year, while their product explained more than $30 \%$ of variation (fig. A1). In contrast, neither of our estimates of fitness predicted genotype frequencies in the third year of the experiment (2009; fig. A1), likely because of the role of unmeasured ecological factors. For example, the very high seedling recruitment in 2008 (including many biennials) created a highly competitive environment, likely one that severely hampered recruitment of new seedlings in 2009. Finally, seed production alone during 2009 predicted genotype frequencies in the final year (2010). Our results echo Harper's argument that accurate predictions of evolutionary change due to natural selection require measuring multiple components of fitness, which should include fecundity and other demographic parameters.

In animal systems, there is reasonably good documentation of how natural selection in the field ultimately results in phenotypic evolution (Morrissey et al. 2010). Assuming that traits are heritable, cases where selection acts on survival (as opposed to siring or fecundity) and where there is no dormancy of progeny (i.e., no seed bank), such as Darwin's finches, are especially likely to show the expected response to selection (Grant and Grant 2008). In plants, most evolutionary studies measure natural selection via fecundity, and very few have directly compared measures of natural selection with realized evolutionary change. Of the five other studies that followed genetic change in plant populations, two are restricted to clonal vegetative reproduction (avoiding the seed-to-adult transitions; Whitlock et al. 2007; Stuefer et al. 2009), and three followed frequency changes of specific alleles in evolving crop populations (Luckett and Edwards 1986; Rhoné et al. 2008; Snow et al. 2010). In one case, where Franks et al. (2007) compared natural selection and the evolutionary response in Brassica populations, there was some concordance between natural selection and evolutionary change, but other demographic filters clearly contributed to fitness. Our experimental results on O. biennis underscore this finding and suggest temporal changes in the importance of fitness components.

\section{Eco-Evolutionary Feedbacks}

Feedbacks between ecology and evolution are being widely discussed, in part because of the realization that evolution can be rapid enough to influence contemporary ecological dynamics (Hairston et al. 2005; Fussmann et al. 2007; Schoener 2011). Our study supports the notion that evolution in plant populations affects concurrent ecological dynamics of interacting herbivore species (Hersch-Green et al. 2011). Indeed, successional species such as ours may be an excellent place to examine eco-evolutionary feedbacks, because the dynamics are nonequilibrial, evolution is likely to be rapid, and the ecology of species interactions may therefore be rapidly reshaped by evolution (Neuhauser et al. 2003). Nonetheless, these same factors, including rapidly changing population densities and community composition, likely also alter eco-evolutionary feedbacks (Neuhauser et al. 2003). In addition, genotype $\times$ environment interactions, which are common, may obscure the detection of an eco- 
evolutionary feedback. In our study of O. biennis, genotypespecific moth attack was correlated between 2007 and 2010 ( $n=16, r=0.661, P=.005)$, which in large measure drove the observed eco-evolutionary feedback (fig. 5). However, we found no such genetic correlation between 2007 and 2009 (or between 2007 and 2008), which likely obscured the eco-evolutionary feedback. Such annual variation in effects is likely to be a challenge in detecting clear signals between evolutionary change and feedbacks to ecology, and it argues for the importance of longer-term studies that link evolution and ecology.

We predict that the benefits of short annual life spans and later flowering will change over time, especially with increased competition. As was previously reported from our single-generation experiments of O. biennis, longer biennial life spans are favored in moderately productive, highly competitive environments (Johnson 2007). Thus, long-term monitoring of our experimental populations may reveal reversals of the current evolutionary trajectories. Given the viability of $O$. biennis seeds over many decades (Darlington 1951) and their requirement for light to germinate (Gross 1985), complex evolutionary dynamics are likely to follow during succession and disturbance. This is especially likely because insect populations, which are affected by life-history evolution and reciprocally affect plant fitness, themselves have temporal dynamics associated with climate and plant density. Thus, not only is $O$. biennis an early-successional species whose life history likely evolves during succession, but we speculate that seed predation may contribute to the maintenance of lifehistory variation because of eco-evolutionary feedbacks.

More generally, rapid evolutionary change has been predicted to alter intraspecific population dynamics (Hairston et al. 2005; Carlson et al. 2011), community composition (Bailey et al. 2009; Johnson et al. 2009b; Palkovacs and Post 2009), and ecosystem processes (Harmon et al. 2009; Bassar et al. 2010). It is the multigenerational feedback of these ecological changes on subsequent evolutionary dynamics that currently awaits testing.

\section{Acknowledgments}

For help with fieldwork, we thank F. Chen, S. Cook-Patton, T. Dodge, A. Erwin, M. Falise, E. Kearney, A. Knight, S. McArt, J. Peters, T. Ramsey, S. Rasmann, A. Smith, M. G. Weber, and E. Woods. Molecular work for this study was conducted in the Evolutionary Genetics Core Facility at Cornell University with support from V. Askinazi, S. Bogdanowicz, R. Harrison, and E. Larson. We thank S. CookPatton, B. Haller, A. Hendry, S. McArt, D. Reznick, R. Shaw, J. Thaler, M. G. Weber, and anonymous reviewers for comments. This study was supported by a U.S. Na- tional Science Foundation (NSF) grant to A.A.A. (EAGER DEB-0950231), which was made possible by the vision of L. Gough. M.T.J.J. was supported by the Natural Sciences and Engineering Research Council of Canada, and J.L.M. was supported by NSF grants DEB-0614406 and DEB0915409.

\section{Literature Cited}

Bailey, J. K., J. A. Schweitzer, F. Úbeda, J. Koricheva, C. J. LeRoy, M. D. Madritch, B. J. Rehill, et al. 2009. From genes to ecosystems: a synthesis of the effects of plant genetic factors across levels of organization. Philosophical Transactions of the Roval Society B: Biological Sciences 364:1607-1616.

Barrett, R. D. H., S. M. Rogers, and D. Schluter. 2008. Natural selection on a major armor gene in threespine stickleback. Science 322:255-257.

Bassar, R. D., M. C. Marshall, A. López-Sepulcre, E. Zandonà, S. K. Auer, J. Travis, C. M. Pringle, et al. 2010. Local adaptation in Trinidadian guppies alters ecosystem processes. Proceedings of the National Academy of Sciences of the USA 107:3616-3621.

Blount, Z. D., C. Z. Borland, and R. E. Lenski. 2008. Historical contingency and the evolution of a key innovation in an experimental population of Escherichia coli. Proceedings of the National Academy of Sciences of the USA 105:7899-7906.

Braak, C. J. F. ter, and P. Šmilauer. 2002. CANOCO reference manual and CanoDraw for Windows user's guide: software for canonical community ordination. Version 4.5. Microcomputer Power, Ithaca, NY.

Brodie, E. D., Jr., B. J. Ridenhour, and E. D. Brodie III. 2002. The evolutionary response of predators to dangerous prey: hotspots and coldspots in the geographic mosaic of coevolution between garter snakes and newts. Evolution 56:2067-2082.

Brown, J. S., and D. L. Venable. 1986. Evolutionary ecology of seedbank annuals in temporally varying environments. American Naturalist 127:31-47.

Carlson, S. M., T. P. Quinn, and A. P. Hendry. 2011. Eco-evolutionary dynamics in Pacific salmon. Heredity 106:438-447.

Carroll, S. P., and C. Boyd. 1992. Host race radiation in the soapberry bug: natural history with the history. Evolution 46:1052-1069.

Cavender-Bares, J., D. D. Ackerly, D. A. Baum, and F. A. Bazzaz. 2004. Phylogenetic overdispersion in Floridian oak communities. American Naturalist 163:823-843.

Charnov, E. L., and W. M. Schaffer. 1973. Life-history consequences of natural selection: Cole's result revisited. American Naturalist 107:791-793.

Cleland, R. E. 1972. Oenothera: cytogenetics and evolution. Academic Press, New York.

Darlington, H. T. 1951. The seventy-year period for Dr. Beal's seed viability experiment. American Journal of Botany 38:379-381.

Ehrlén, J. 2003. Fitness components versus total demographic effects: evaluating herbivore impacts on a perennial herb. American Naturalist 162:796-810.

Franks, S. J., S. Sim, and A. E. Weis. 2007. Rapid evolution of flowering time by an annual plant in response to a climate fluctuation. Proceedings of the National Academy of Sciences of the USA 104: $1278-1282$.

Fussmann, G. F., M. Loreau, and P. A. Abrams. 2007. Eco-evolu- 
tionary dynamics of communities and ecosystems. Functional Ecology 21:465-477.

Garland, T., Jr., and M. R. Rose. 2009. Experimental evolution: concepts, methods, and applications of selection experiments. University of California Press, Berkeley.

Grant, P. R., and B. R. Grant. 2008. How and why species multiply. Princeton University Press, Princeton, NJ.

Gross, K. L. 1985. Effects of irradiance and spectral quality on the germination of Verbascum thapsus L. and Oenothera biennis L. seeds. New Phytologist 101:531-541.

Hairston, N. G., S. P. Ellner, M. A. Geber, T. Yoshida, and J. A. Fox. 2005. Rapid evolution and the convergence of ecological and evolutionary time. Ecology Letters 8:1114-1127.

Harmon, L. J., B. Matthews, S. Des Roches, J. M. Chase, J. B. Shurin, and D. Schluter. 2009. Evolutionary diversification in stickleback affects ecosystem functioning. Nature 458:1167-1170.

Harper, J. L. 1977. Population biology of plants. Academic Press, London.

Hart, R. 1977. Why are biennials so few. American Naturalist 111: 792-799.

Hersch-Green, E. I., N. E. Turley, and M. T. J. Johnson. 2011. Community genetics: what have we accomplished and where should we be going? Philosophical Transactions of the Roval Society B: Biological Sciences 366:1453-1460.

Jain, S. K. 1982. Variation and adaptive role of seed dormancy in some annual grassland species. Botanical Gazette 143:101-106.

Johnson, M. T. J. 2007. Genotype-by-environment interactions leads to variable selection on life-history strategy in common evening primrose (Oenothera biennis). Journal of Evolutionary Biology 20: 190-200.

- 2011. The contribution of evening primrose (Oenothera biennis) to a modern synthesis of evolutionary ecology. Population Ecology 53:9-21.

Johnson, M. T. J., and A. A. Agrawal. 2005. Plant genotype and environment interact to shape a diverse arthropod community on evening primrose (Oenothera biennis). Ecology 86:874-885.

Johnson, M. T. J., A. A. Agrawal, J. L. Maron, and J. P. Salminen. $2009 a$. Heritability, covariation and natural selection on 24 traits of common evening primrose (Oenothera biennis) from a field experiment. Journal of Evolutionary Biology 22:1295-1307.

Johnson, M. T. J., M. Vellend, and J. R. Stinchcombe. 2009b. Evolution in plant populations as a driver of ecological changes in arthropod communities. Philosophical Transactions of the Roval Society B: Biological Sciences 364:1593-1605.

Kinnison, M. T., and A. P. Hendry. 2001. The pace of modern life II: from rates of contemporary microevolution to pattern and process. Genetica 112-113:145-164.

Lacey, E. P., L. Real, J. Antonovics, and D. G. Heckel. 1983. Variance models in the study of life histories. American Naturalist 122:114131.

Larson, E. L., S. M. Bogdanowicz, A. A. Agrawal, M. T. J. Johnson, and R. G. Harrison. 2008. Isolation and characterization of polymorphic microsatellite loci in common evening primrose (Oenothera biennis). Molecular Ecology Resources 8:434-436.

Law, R., A. D. Bradshaw, and P. D. Putwain. 1977. Life-history variation in Poa annua. Evolution 31:233-246.
Losos, J. B. 1994. Integrative approaches to evolutionary ecology: Anolis lizards as model systems. Annual Review of Ecology and Systematics 25:467-493.

Luckett, D. J., and K. J. R. Edwards. 1986. Esterase genes in parallel composite cross barley populations. Genetics 114:289-302.

Maron, J. L., M. Vilà, R. Bommarco, S. Elmendorf, and P. Beardsley. 2004. Rapid evolution of an invasive plant. Ecological Monographs 74:261-280.

Meyer, J. R., and R. Kassen. 2007. The effects of competition and predation on diversification in a model adaptive radiation. Nature 446:432-435.

Mooney, K. A., R. Halitschke, A. Kessler, and A. A. Agrawal. 2010. Evolutionary trade-offs in plants mediate the strength of trophic cascades. Science 327:1642-1644.

Morrissey, M. B., L. E. B. Kruuk, and A. J. Wilson. 2010. The danger of applying the breeder's equation in observational studies of natural populations. Journal of Evolutionary Biology 23:2277-2288.

Neuhauser, C., D. A. Andow, G. E. Heimpel, G. May, R. G. Shaw, and S. Wagenius. 2003. Community genetics: expanding the synthesis of ecology and genetics. Ecology 84:545-558.

Palkovacs, E. P., and D. M. Post. 2009. Experimental evidence that phenotypic divergence in predators drives community divergence in prey. Ecology 90:300-305.

Rauwolf, U., H. Golczyk, J. Meurer, R. G. Herrmann, and S. Greiner. 2008. Molecular marker systems for Oenothera genetics. Genetics 180:1289-1306.

Reznick, D. A., H. Bryga, and J. A. Endler. 1990. Experimentally induced life-history evolution in a natural population. Nature 346 : 357-359.

Rhoné, B., C. Remoué, N. Galic, I. Goldringer, and I. Bonnin. 2008. Insight into the genetic bases of climatic adaptation in experimentally evolving wheat populations. Molecular Ecology 17:930-943.

Schoener, T. W. 2011. The newest synthesis: understanding the interplay of evolutionary and ecological dynamics. Science 331:426-429.

Smith, J. W., and C. W. Benkman. 2007. A coevolutionary arms race causes ecological speciation in crossbills. American Naturalist 169: 455-465.

Snow, A. A., T. M. Culley, L. G. Campbell, P. M. Sweeney, S. G. Hegde, and N. C. Ellstrand. 2010. Long-term persistence of crop alleles in weedy populations of wild radish (Raphanus raphanistrum). New Phytologist 186:537-548.

Stuefer, J. F., N. P. R. Anten, H. de Kroon, J. L. Peters, A. SmitTiekstra, P. J. Vermeulen, and H. J. During. 2009. Genotypic selection shapes patterns of within-species diversity in experimental plant populations. Journal of Ecology 97:1020-1027.

Waser, N. M., D. R. Campbell, M. V. Price, and A. K. Brody. 2010. Density-dependent demographic responses of a semelparous plant to natural variation in seed rain. Oikos 119:1929-1935.

Whitlock, R., J. P. Grime, R. Booth, and T. Burke. 2007. The role of genotypic diversity in determining grassland community structure under constant environmental conditions. Lournal of Ecology 95: 895-907. 